\title{
Penoserotal Lymphedema Secondary to Hidradenitis Suppurativa
}

Tlili Ghassen, Jaidane Mehdi, Bouassida Khaireddine, Mosbah Ali Tahar

Department of Urology, Hospital of Sahloul, Sousse, Tunisia.

Abstract:

Hidradenitis suppurativa is a chronic, recurrent inflammatory disease affecting the cutaneous apocrine glands and resulting in their obstruction [1]. This enigmatic disease causes recurrent episodes of infection, edema, scarring, and fibrosis of surrounding tissues. We present the unusual case of a 58-year-old white male with fifteen years of hidradenitis suppurativa (HS) complicated by the occurrence of penoscrotal elephantiasis.

Key words: Hidradenitis Suppurativa, Elephantiasis, Edema, Scrotum, Cicatrix.

Introduction

Hidradenitis suppurativa (HS) is a chronic, recurrent inflammatory disease affecting the cutaneous apocrine glands and resulting in their obstruction [1]. Massive scrotal lymphedema, or elephantiasis, has been described in patients with chronic inflammation of the inguinoscrotal region, filariasis, and following inguinal irradiation and/or lymphadenectomy [2]. The association of elephantiasis and hidradenitis suppurativa is not well recognized. We found the mention of penoscrotal lymphedema caused by HS to be of particular interest and would like to share the observation of a patient who developed an unusual complication of penoscrotal elephantiasis caused by longstanding HS.

\section{Case Report}

A 58-year-old white male with no recent travel history was identified with scrotal enlargement that began about 15 years ago but had become notably worse over the past 2 years. There was no history of overseas travel prior to surgery, or irradiation. The patient was treated previously for hidradenitis suppurativa with antibiotics, but it was not effective. The physical examination revealed a massively enlarged, indurated scrotum that obscured his penis and had multiple sinus tracts draining clear and foulsmelling fluid. The testes and cord structures were not palpable, and there was no obvious regional lymphadenopathy. The perineum was grossly indurated and scarred [Fig.1]. The diagnosis of scrotal elephantiasis secondary to HS with multiple episodes of lymphatic scarring was made.

The patient underwent wide excision of the scrotum and perineum. Once in the lithotomy position, a Foley catheter was placed, and a short vertical incision was made through the skin and subcutaneous tissue to liberate the whole length of the penis. The scrotal mass was then excised using

\section{Corresponding Author: Dr. Bouassida Khaireddine}

Email: khayri14@hotmail.com

Received: September 29, 2014 | Accepted: April 18, 2015 | Published Online: May 10, 2015

This is an Open Access article distributed under the terms of the Creative Commons Attribution License (creativecommons.org/licenses/by/3.0)

Conflict of interest: None declared | Source of funding: Nil | DOl: http://dx.doi.org/10.17659/01.2015.0050 
two curvilinear incisions. The first incision was made with an upper convexity at the root of the scrotum, and the second with its upper concavity at the limit of the healthy skin of the posterior perineum. The elephantoid mass was excised [Fig.2]. The testes appeared normal, but there were small hydroceles. The hydroceles were repaired by the Jaboulay technique. Healthy skin with underlying soft tissue was mobilized from the sides and advanced medially to cover the testes and the spermatic cords. Midline suturing of this scrotal skin flap was done so that it simulated the appearance of the median raphe. Scrotoplasty procedure was completed by inserting two closed suction drains in the scrotal sac [Fig.3].

\section{Discussion}

Elephantiasis has been a recognized complication of inguinal node irradiation, filarial infection, tumor

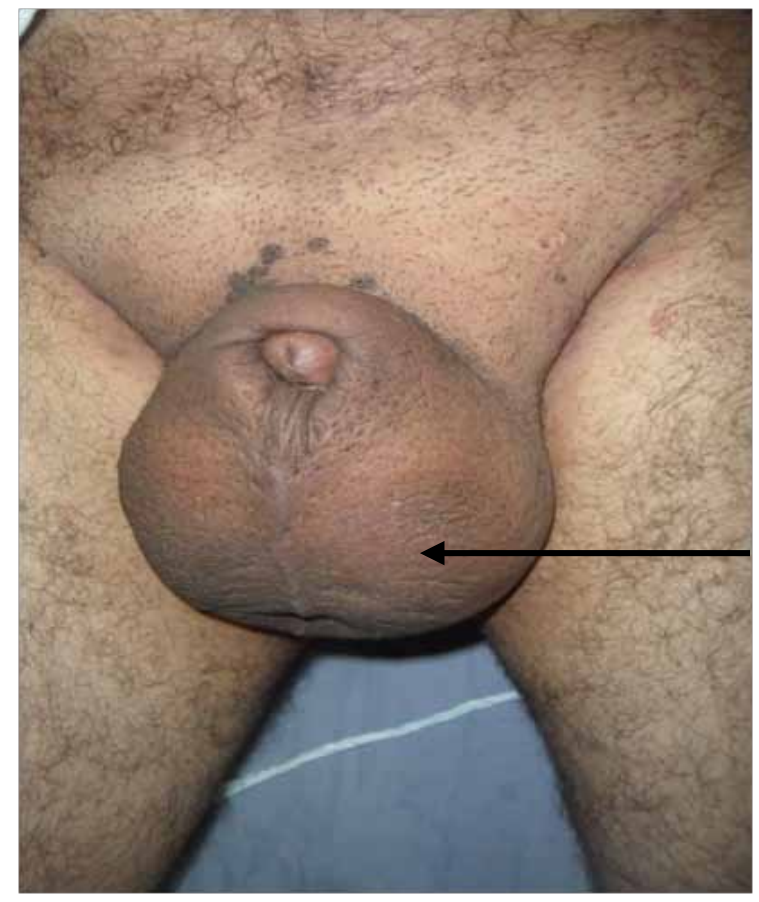

Fig.1: Massive scrotal edema with draining sinus tracts in patient with chronic hidradenitis suppurativa (arrow).

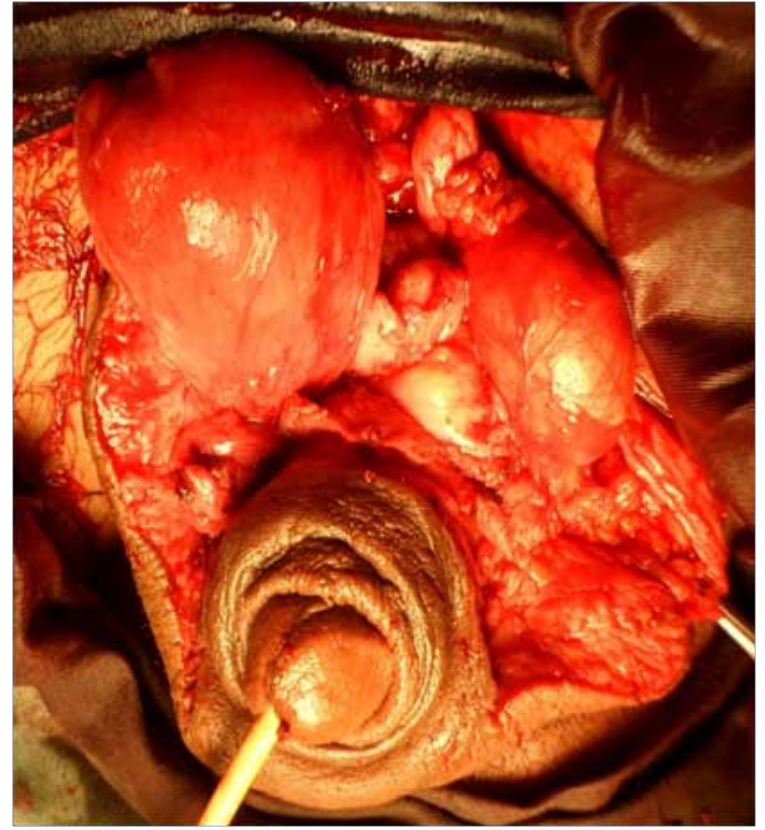

Fig.2: Excision of diseased scrotal skin extended in lateral and posterior directions until healthy skin encountered.

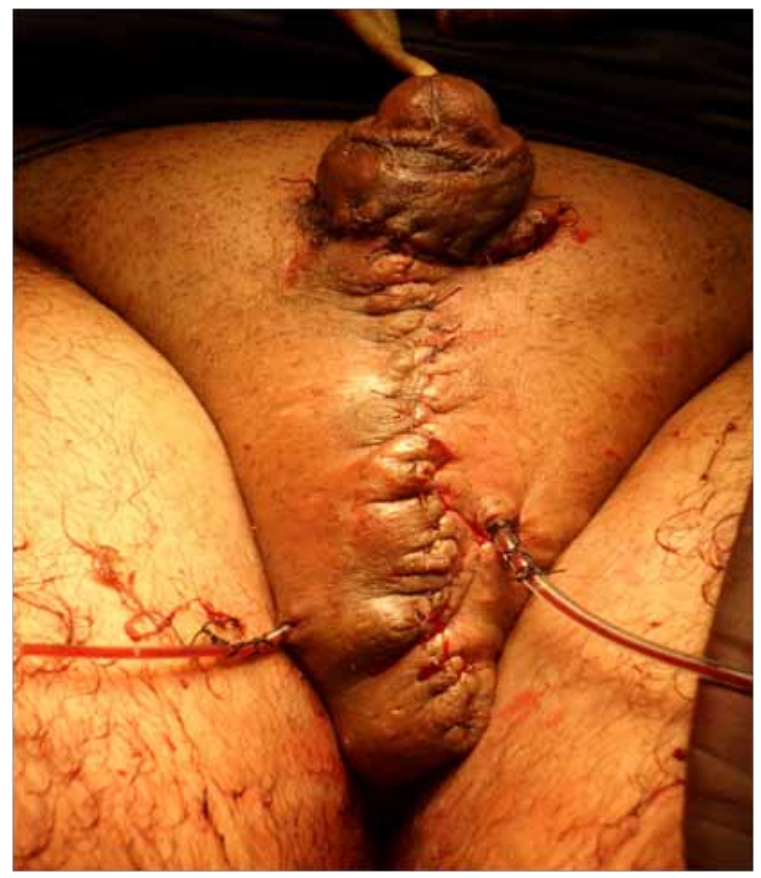

Fig.3: Immediate result after surgery. 
involvement, and surgical lymphatic destruction, but it has only been reported in association with HS on a few occasions [3]. Matas defines elephantiasis as a progressive histopathologic state characterized by a chronic inflammatory fibromatosis or hypertrophy of the hypodermal and dermal connective tissue, preceded by, and associated with lymphatic venous stasis [4]. The histopathologic elements are: mechanical obstruction or blockage of the veins and lymphatics generally caused by thrombophlebitis, lymphangitis or adenitis, hyperplasia of the collagenous connective tissue of the hypodermis, the gradual disappearance of the elastic fibers of the skin, the presence of a coagulable dropsy or hard lymphedema, and chronic reticular lymphangitis due to secondary and repeated invasion by pathogenic micro-organisms of the streptococcal type [5].

HS is a chronic inflammatory and infectious disease of unknown etiology, commonly affecting the skin in the axillary, inguinal, and perianal regions [1]. The etiology of hidradenitis suppurativa is thought to be a blockage of the ducts of the apocrine glands by secretions, with consequent ductal dilatation, infection, and abscess formation. Staphylococcus is the most common infecting organism, but other gram-positive organisms and micrococci are found occasionally [5].

Various conservative treatment measures have been adopted for hidradenitis suppurativa with uniformly poor results. Radiation, antibiotic combinations, and 13-cis-retinoic acid have all yielded suboptimal results. Antistaphylococcal vaccines have been used in these patients on the premise that they can correct the presumed defective immunologic response to staphylococci [6]. For advanced stages, surgical therapy often remains the only solution. Microsurgical procedures include lymph angioplasty or lymphatic venous anastomosis and have proved effective in some cases [7]. In extensive disease, usually only complete excision of all elephantoid tissue, preferably saving the penis, spermatic cord, and testes, is appropriate. Plastic penoscrotal reconstruction must be attempted [8].

The skin of the scrotal neck has been the best to reconstruct the scrotum and to accommodate the testicular structures. Different techniques such as posterior and ventral pedunculated scrotal flaps and split skin grafting to the penis have proved successful. Dandapat et al. published results of the only large group of 350 patients with elephantiasis of the penis and scrotum who underwent complete excision and penoscrotal reconstruction [9].

\section{Conclusion}

Hidradenitis suppurativa is a chronic relapsing infection of the apocrine sweat glands. Its association with penoscrotal lymphedema is not well recognized. Many techniques have been described for the treatment of penile and scrotal elephantiasis. Our modification of the BotreauRoussel [10] and Feins [11] techniques in the length and the orientation of the incision lines have given satisfactory results in the covering procedures, with excellent final cosmetic results.

\section{References}

1. Baughman SM, Cespedes RD. Unusual presentation of hidradenitis suppurativa with massive enlargement of penis. Urology. 2004;64:377-378.

2. Konety BR, Cooper T, Flood HD, Futrell JW. Scrotal elephantiasis associated with hidradenitis suppurativa. Plast Reconstr Surg. 1996;97:1 243-1245.

4. Alikhan A, Lynch PJ, Eisen DB. Hidradenitis suppurativa: acomprehensive review. J Am Acad Dermatol. 2009;60:539-561.

5. Stryker GV, Ploch B. Elephantiasis of the penis and scrotum, a sequela of lymphogranuloma inguinale. Arch Dermat. 1935;32:86-89.

6. Anderson DK, Perry AW. Axillary hidradenitis. 
Arch. Surg. 1975;1 10:69-72.

7. Kress DW, Graham WP III, Davis TS, Miller SH. A preliminary report on the use of Staphage Lysate for treatment of hidradenitis suppurativa. Ann. Plast. Surg. $1981 ; 6: 393-395$.

8. Huang GK. Microsurgical therapy of scrotum elephantiasis. Z Urol Nephrol. 1989;82:459462.

9. Apesos J, Anigian G. Reconstruction of penile and scrotal lymphedema. Ann Plast Surg.
$1991 ; 27: 570-573$.

10. Kuepper D. Giant scrotal elephantiasis. Urology. 2005;65:389.

11. Ndoye $A 1$, Sylla $C, B a$, Guèye SM, Diagne BA. Point of technique: Management of penile and scrotal elephantiasis. BJU Int. 1999;84:362364.

12. Feins NR. A new incision for penile surgery. J Ped Surg. $1981 ; 16: 817-819$. 